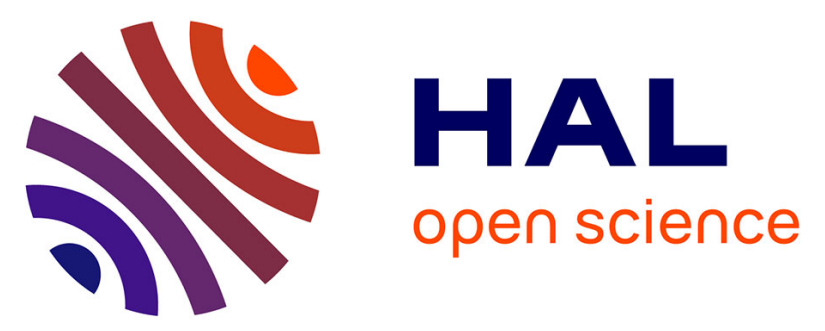

\title{
Ni-based superalloy: crystalline orientation mapping and gamma - gamma' phases discrimination with the iCHORD method
}

Cyril Langlois, Marie-Agathe Charpagne, Suzanne Vernier, Sébastien Dubail, Thierry Douillard, Nathalie Bozzolo

\section{To cite this version:}

Cyril Langlois, Marie-Agathe Charpagne, Suzanne Vernier, Sébastien Dubail, Thierry Douillard, et al.. Ni-based superalloy: crystalline orientation mapping and gamma - gamma' phases discrimination with the iCHORD method. European Microscopy Congress, Aug 2016, Lyon, France. 10.1002/9783527808465.emc2016.6924 . hal-02138404

\author{
HAL Id: hal-02138404 \\ https://hal.science/hal-02138404
}

Submitted on 24 May 2019

HAL is a multi-disciplinary open access archive for the deposit and dissemination of scientific research documents, whether they are published or not. The documents may come from teaching and research institutions in France or abroad, or from public or private research centers.
L'archive ouverte pluridisciplinaire HAL, est destinée au dépôt et à la diffusion de documents scientifiques de niveau recherche, publiés ou non, émanant des établissements d'enseignement et de recherche français ou étrangers, des laboratoires publics ou privés. 


\section{Ni-based superalloy: crystalline orientation mapping and $\gamma-\gamma^{\prime}$ phases discrimination with the iCHORD method}

\author{
C. Langlois ${ }^{1}$, M-A. Charpagne ${ }^{2}$, S. Vernier ${ }^{2}$, S. Dubail ${ }^{3}$, \\ T. Douillard ${ }^{1}$, N. Bozzolo²
}

${ }^{1}$ University of Lyon, MATEIS, umr CNRS 5510, Bât. B. Pascal, INSA de Lyon/Université Lyon I, 69621 Villeurbanne Cedex, France ${ }^{2}$ CEMEF - Mines ParisTech, umr CNRS 7635, CS 10207 rue Claude Daunesse 06904 Sophia Antipolis Cedex, France ${ }^{3}$ AXON SQUARE SAS, 4 la Tuilière, 74140 SCIEZ, France

\section{Ni-based superalloy: $\gamma-\gamma^{\prime}$ orientation mapping}

Electron backscatter diffraction (EBSD) is routinely employed as a characterization tool to obtain individual grain orientations, local texture and phase identification. However, in the case of $\gamma-\gamma$ Ni-based superalloys, the EBSD technique allows mapping the orientations but fails discriminatin the two phases because their diffraction signature is too similar. A coupling with EDX analysis for instance helps to identify the two phases but suffers from the lack of spatial resolution of the EDX maps. Another way to discriminate the two phases is to use the BSE images, sensible to the chemistry, but because of the difference in the geometry of acquisition of the EBSD images and the BSE images, superposition of the two information is complicated by strong spatial distortions. In this context, any new technique that can lead to an easier phase and orientation mapping would be welcome, especially to resolve the fine secondary $\gamma^{\prime}$ precipitates (typically few tens of How to efficiently discriminate between $\gamma$ matrix and $\gamma^{\prime}$ precipitates in an orientation map?

\section{Orientation mapping using channeling}

Here is a brief summary of the iCHORD technique, allowing obtaining orientation maps in FIB microscope without the need of an EBSD camera.

\section{Image acquisition}

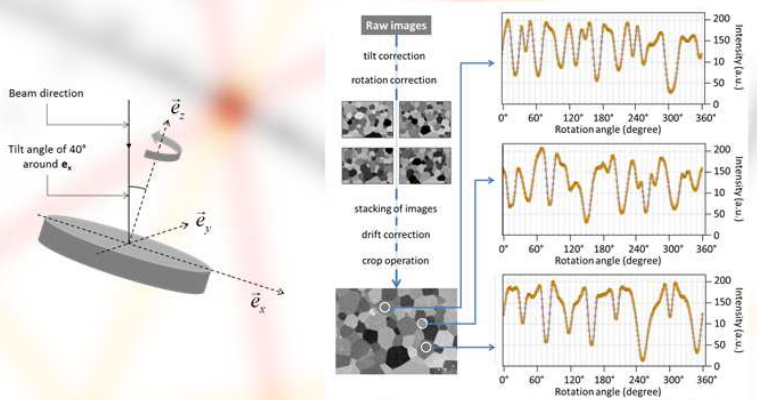

The sample is first tilted to an angle of $40^{\circ}$. Then, it is rotated around its tilted normal. For each rotation step, an image is acquired using the secondary electron detector. A complete $360^{\circ}$ rotation is achieved, resulting in a series of ion images.

All these images are then corrected from the $40^{\circ}$ tilt and for the rotation, in order to have the region of interest oriented the same way in all the images.

All the images are then stacked together and the residual drift is corrected. At the end intensity profiles can be extracted by plotting the intensity of each pixel along the stack, at given $(\mathrm{X}, \mathrm{Y})$ position in all the images.

\section{Theoretical database construction}
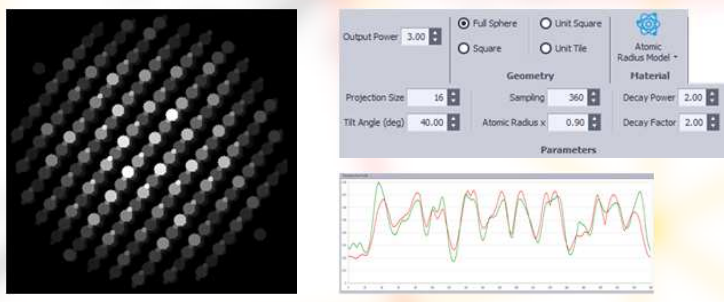

For a given orientation of a crystal (i.e. one grain in one image of the series), the intensity received by the detector from this crystal can be related to the projection of the atoms on a plane corresponding to the sample surface. More precisely, it is the sum of the grey levels of the pixels composing the projection that can be related to the intensity received, after some mathematical adjustments for the relative contribution of each atom in the projection. It constitutes one data point in an intensity profile. The orientation of the crystal is then changed the same way as the experimental conditions, and the complete theoretical profile is obtained. The database is then constructed by sampling the orientation space, and associating an intensity profile to each Euler triplet. As we are in a cubic system, the Euler angles vary between $0^{\circ}$ and $360^{\circ}$ for $\varphi_{1}$, and between $0^{\circ}$ and $90^{\circ}$ for $\phi$ and $\varphi_{2}$ (Bunge convention). Euler angles are converted to quaternions for simplicity sakes in the computations.

\section{Database search}

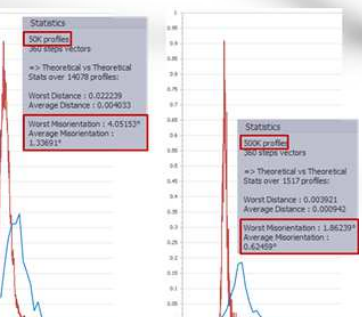

The search in the database starts with some " brute force " search for a certain amount of positions in the area. Then, an algorithm relying on similarities between the neighbouring profiles is used to index the pixels.

The size of the database increases the angular resolution but also the indexatio time. However, it is possible to statistically evaluate the angular resolution obtained with a given database size. It is then the user that choose which precision is required for a given application.
Mixing EBSD, BSE and EDS maps : not convincing
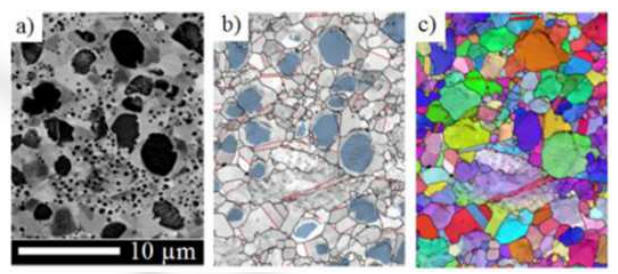

(a) BSE image

(b) EDS resolved phase map

Orientation map with a color code indicating which of the scanned section (see standard triangle)

\section{${ }_{001} A_{101}^{111}$}

The above figure shows the chemical and structural data that can be obtained on the same area of a Ni-based superalloy. The EBSD data (c) are obtained with a sample tilted to $70^{\circ}$ whereas the BSE images (a) are obtained with the normal of the sample perpendicular to the incident electron beam. BSE and EBSD maps are therefore difficult to superimpose because of image distorsions due to the different geometries of acquisition. Concerning EDS maps, the interaction volume is very large compared to the electron probe. The actual resolution of the EDS maps is then about $1 \mu \mathrm{m}$. This coarse spatial resolution prevents detecting the smallest $\gamma^{\prime}$ precipitates in correlation to EBSD (b).

\section{Orientation maps using iCHORD on a Ni-based superalloy}
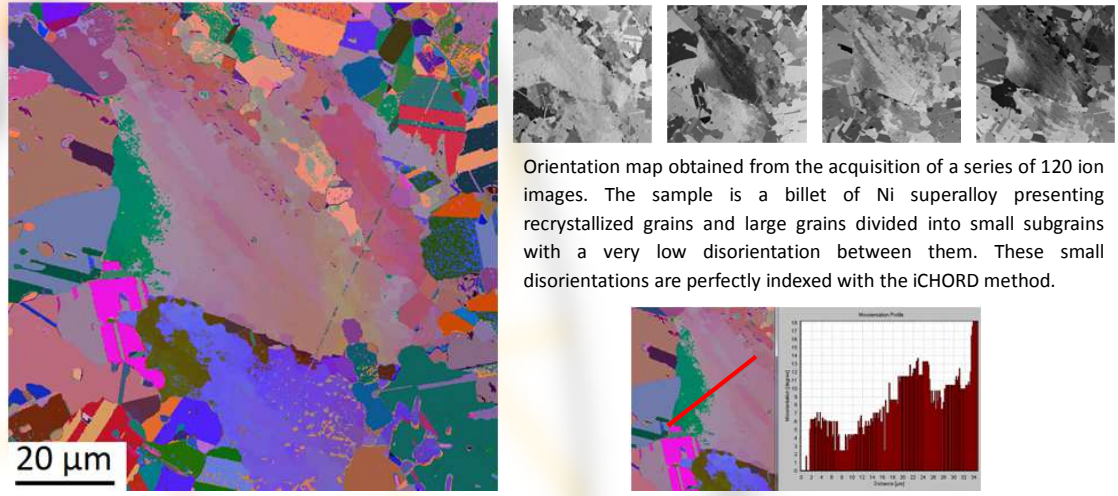

Orientation map obtained from the acquisition of a series of 120 ion images. The sample is a billet of $\mathrm{Ni}$ superalloy presenting recrystallized grains and large grains divided into small subgrains with a very low disorientation between them. These small disorientations are perfectly indexed with the iCHORD method.

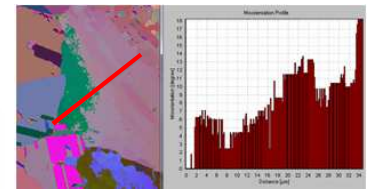

\section{$\gamma-\gamma^{\prime}$ discrimination using secondary ion images}

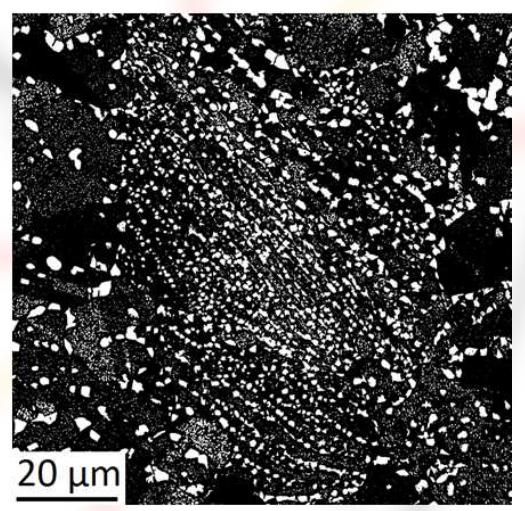

detector used for the acquisition of mages series can also be used to detect secondary ions, by changing its polarity. The resulting images present a strong contrast between $\gamma$ and $\gamma$ phases, with also a bit of channeling contrast. To remove the channeling information, several images are acquired at different orientation (during the rotation series) and an average image is calculated. A threshold operation can then be applied to this average image, with no more channeling contrast.

On the image presented here, three families of precipitates are present with different size ranges. In the recrystallized area, large precipitates of 3-5 $\mu \mathrm{m}$ are visible near the grain boundaries as well as very small precipitates inside the grains. In the deformed area (in the center), precipitates with $\sim 1 \mu \mathrm{m}$ diameter are visible.

\section{Mixing the information}

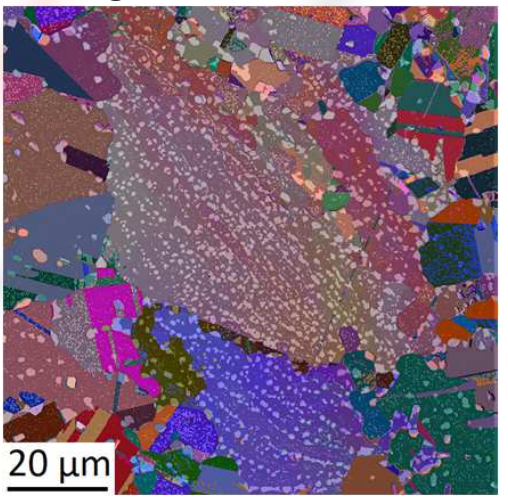

Because the secondary ion images are taken using the same detector at the same position, and the same incident beam and scan system, there is almost no distorsions between the secondary electron images and the secondary ion image. As a consequence, the final orientation map and the secondary in average image can be superimposed with only a small drift correction.

From the superimposed image, we can read both the orientation and the phase, which impossible when the EBSD used alone without any complementary technique, and quite difficult when the EBSD technique is used together with EDS spectra or BSE images.

Conclusion Using the iCHORD method allows obtaining orientation maps with a very good spatial resolution on $\gamma-\gamma$ sample (pixel size $0.14 \mu \mathrm{m}$ ). Mixing the orientation map with chemical information obtained with secondar ion images allows discriminating between the two phases, keeping the spatial resolution. 